\title{
Reducing Spectrum Handoffs and Energy Switching Consumption of MADM-Based Decisions in Cognitive Radio Networks
}

\author{
Rafael Aguilar-Gonzalez, ${ }^{1,2}$ Marco Cardenas-Juarez, ${ }^{1}$ Ulises Pineda-Rico, ${ }^{1}$ Armando Arce, ${ }^{3}$ \\ Matti Latva-aho, ${ }^{2}$ and Enrique Stevens-Navarro ${ }^{1}$ \\ ${ }^{1}$ Facultad de Ciencias, Universidad Autónoma de San Luis Potosí (UASLP), Av. Salvador Nava Mtz. s/n, Zona Universitaria, \\ 78290 San Luis Potosí, SLP, Mexico \\ ${ }^{2}$ Centre for Wireless Communications, University of Oulu, P.O. Box 4500, 90014 Oulu, Finland \\ ${ }^{3}$ Instituto Tecnológico de Sonora, 5 de Febrero 818, Col. Centro, 85000 Ciudad Obregón, SON, Mexico \\ Correspondence should be addressed to Enrique Stevens-Navarro; estevens@galia.fc.uaslp.mx
}

Received 5 February 2016; Revised 22 October 2016; Accepted 1 November 2016

Academic Editor: Francesco Gringoli

Copyright (C) 2016 Rafael Aguilar-Gonzalez et al. This is an open access article distributed under the Creative Commons Attribution License, which permits unrestricted use, distribution, and reproduction in any medium, provided the original work is properly cited.

\begin{abstract}
In a cognitive radio network (CRN), the number of spectrum handoffs increases energy consumption of cognitive (or secondary) users due to the channel switching process. This might limit the operation of the CRN, especially in scenarios where secondary users terminals are battery-powered. Thus, reducing the number of times a cognitive user involved in a transmission switch to different spectrum holes is required to increase battery life-time. In this regard, available spectrum holes possess different attributes (e.g., bandwidth) that can be exploited to satisfy specific secondary users requirements (i.e., connection profile) for data transmission while saving energy. Here, three multiple attribute decision-making (MADM) algorithms for the spectrum decision functionality are evaluated using real spectrum measurements of TV bands. This is performed by proposing six decision parameters, which are extracted from the spectrum data to characterize its suitability. Then, these are used as inputs of the MADM algorithms to select the most suitable spectrum hole for a cognitive user. Thus, an enhanced MADM-based decision process is proposed to reduce the number of handoffs considering energy consumption due to channel switching (ECCS). Results quantify savings from $30 \%$ to $90 \%$ in ECCS and spectrum handoffs reductions from $47 \%$ to $90 \%$.
\end{abstract}

\section{Introduction}

Cognitive radio networks (CRNs) are envisioned to cope with the spectrum scarcity problem of future wireless networks $[1,2]$. Moreover, recent trends have shown the need for developing novel energy-efficient cognitive functions and abilities to enable green CRNs $[3,4]$. Among the main functions of a Cognitive Radio (CR) device, spectrum sensing and spectrum decision play key roles at supporting the envisioned opportunistic spectrum access. In such scenario, by performing the spectrum sensing function, an unlicensed user with a CR device or secondary user (SU) must sense first the surrounding radiofrequency (RF) environment to determine unused portions of the spectrum (i.e., spectrum holes) from licensed spectrum users or primary users
(PUs). Then, through the spectrum decision function, the CR device should select the best spectrum hole for its opportunistic transmission either by itself or assisted by the network.

Once a hole is selected, a spectrum handoff shall be executed. Hence, the RF front-end of the CR device requires several energy-consuming reconfiguration procedures to be able to transmit in the selected frequency. Indeed, this is known in the literature as the energy consumption due to channel switching (ECCS) [5]. Additionally, other problems may arise if the spectrum decision algorithm tends to select different spectrum holes very frequently; for example, the unwanted ping-pong effect may appear thus affecting SUs connection quality $[6,7]$. Therefore, an adequate spectrum decision should take into account the intrinsic tradeoff 


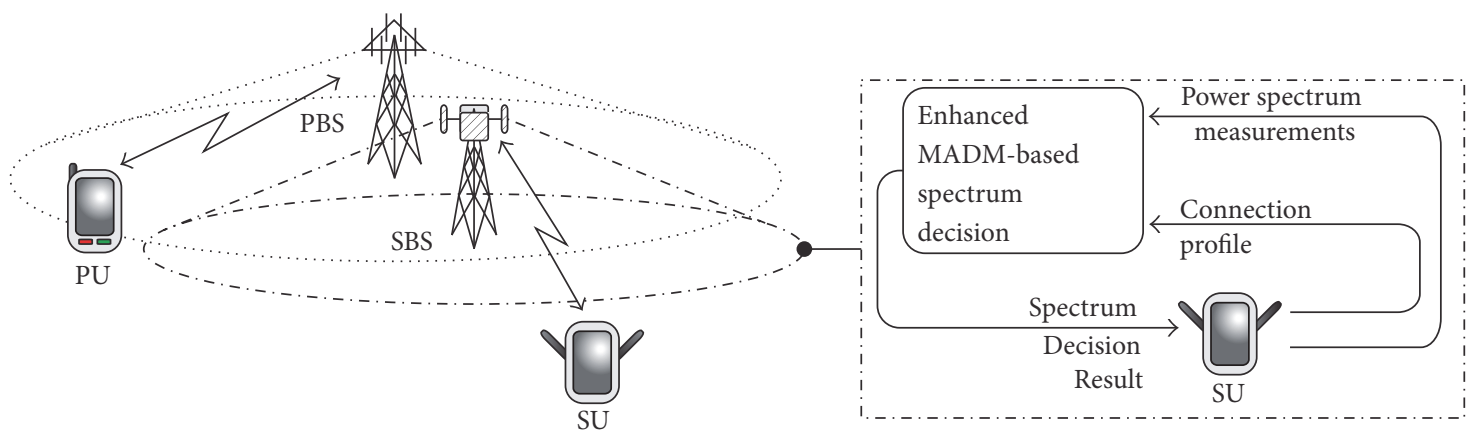

PBS: primary base station SBS: secondary base station

PU: primary user SU: secondary user

FIGURE 1: System model for the EMSD functionality.

among handoff execution and its related energy consumption.

Selecting the best frequency band for a given transmission requires making preference decisions over the set of available alternatives of spectrum holes, which are indeed characterized by different attributes. Therefore, spectrum decision can be modeled as a multiple attribute decisionmaking (MADM) problem [8]. MADM algorithms have been previously utilized for network selection in the context of heterogeneous wireless networks [9-11]. Moreover, these algorithms have been also considered for the spectrum decision function in CRNs. Results on this matter have been presented in [12], where an Analytical Hierarchy Process (AHP) was utilized to select available frequency bands in CRNs. Furthermore, in earlier stages of this investigation [13, 14], we evaluated the performance of three MADM decision algorithms: Simple Additive Weighting (SAW), Technique for Order Preferences by Similarity to Ideal Solution (TOPSIS), and the Compromise Ranking Method VIKOR using real spectrum occupancy measurements in a practical scenario.

In this paper, we propose an Enhanced MADM-based Spectrum Decision (EMSD) functionality for CRNs. In this regard, available spectrum holes possess different attributes (e.g., bandwidth size, occupancy, and central frequency) that can be taken into account for the spectrum decision. Also, the selected spectrum holes shall satisfy specific secondary users requirements (i.e., connection profile) for data transmission while trading off energy consumption. Here, the abovementioned MADM algorithms for the spectrum decision functionality are evaluated using real spectrum usage information of TV bands. This is performed by considering six decision parameters, which are extracted from the spectrum data to characterize its suitability. Then, these are used as inputs of the MADM algorithms to select the most suitable spectrum hole for a cognitive user while reducing the number of spectrum handoffs considering ECCS.

This paper is organized as follows. Section 2 describes the system model as well as the six decision parameters considered. The MADM algorithms are presented in Section 3. Section 4 introduces the EMSD functionality. Results are shown and discussed in Section 5. Finally, Section 6 concludes this paper.

\section{System Model for Spectrum Decision}

Let us consider a wireless networking scenario consisting of a primary centralized network coexisting with a secondary centralized network implementing CR techniques in the same geographical area as shown in Figure 1. Thus, SUs follow a network-assisted spectrum decision and hence a network-assisted spectrum handoff approach. The SUs execute power spectrum measurements periodically. The secondary base station (SBS) concentrates the two main data types generated by SUs: power spectrum measurements and connection profile. A set of prestored connection profiles are assumed and they can be selected by the SUs based on its connection QoS requirements. For example, for a data intensive connection requirement, a specific bandwidth-oriented connection profile is selected to match such requirements. Thus, the connection profile aims to match the connection requirements to the spectrum decision process. SUs and SBS are connected through a dedicated control channel. All the obtained information is sent back to the network, where it is processed in the proposed Enhanced MADM-based Spectrum Decision (EMSD) function and sent back to the SBS, which delivers the results to each SU spectrum decision. The system model proposed can be implemented by means of specific purposes equipment at the SBS or implemented in cutting-edge network technologies such as cloud cognitive networks [15-17].

The spectrum decision process begins with the feeding of the EMSD function with corresponding spectrum measurements (i.e., samples of the spectrum power) and connection profile according to QoS requirements. Internally, the EMSD function computes six decision parameters to describe each spectrum hole available. Thus, the spectrum decision is formulated as a MADM problem with several alternatives (i.e., spectrum holes) and each one is described by a set of decision parameters (e.g., bandwidth size). Then, the MADM algorithms use this information and select the best spectrum hole. Finally, the decision is traded off for the energy consumption required to execute the spectrum handoff.

2.1. Decision Parameters. To start the spectrum decision process, the available spectrum holes have to be identified. 
Then, for each spectrum hole a set of parameters describing its features or characteristics have to be calculated. Thus, spectrum usage information is required to both identify the holes and calculate its features. Within the EMSD function, the information coming from spectrum usage is processed to characterize each spectrum hole. In a real system this process should be done according to PU activity and also based on SU connection requirements. Each time a connection requirement occurs, a spectrum decision should be executed to satisfy SU connection requirements. Once the connection is established in a specific spectrum hole, then periodic spectrum decisions have to be considered based on the availability of the current spectrum hole, connection requirements, and trading off energy consumption due to frequent spectrum handoffs (i.e., ping-pong effect). However, to increase the certainty of the exact moment for making a spectrum decision, sensing and statistical information need to be combined [18]. Finally, it is worth mentioning that the spectrum measurements can be affected by the presence of hidden nodes transmissions. To overcome this issue, a cooperative spectrum sensing approach has to be considered. For example, data fusion centers may be deployed in the SBSs to help overcome these scenarios as proposed in [19]. However, for the scope of this manuscript the cooperative spectrum sensing scenario is not considered.

In this work, for each spectrum decision interval, a specific number of spectrum samples are obtained from spectrum measurements executed periodically in the SU terminal. This spectrum information is assigned into a matrix called interval decision ID $_{n}$, with $n=1, \ldots, N$, and where $N$ is the total number of consecutive spectrum decisions. The ID $_{n}$ matrix is expressed in (1). Thereby, every time a spectrum decision is made, the total number of spectrum samples analyzed during the decision interval is $S$, with $s=1, \ldots, S$, and the total number of resolution points is $P$ corresponding to the examined frequencies, with $p=1, \ldots, P$. Here, it is important to mention the existence of the auxiliary vector $\mathbf{f} \mathbf{v}$ that has the frequency value of each $p$ resolution point and that is used as identifier. Thus, $\mathrm{id}_{s, p}$ is any $\mathbf{I D}_{n}$ element that has a sample of the spectral power in a frequency point:

$$
\mathbf{I D}_{n}=\left[\mathrm{id}_{s, p}\right]_{S \times P}
$$

It is worth mentioning that the spectrum sensing capabilities are in fact limited by the SU hardware capabilities which may vary from device to device. We assume that homogeneous CR devices are used in our scenario. This assumption may be valid for early deployments of CRNs. However, as these networks grow in deployments and in number of users, the homogeneity among CR devices will not be valid. Also, in case of missing frequency samples, the system may be delayed to obtain more samples or previous samples available may be used temporarily. In both cases, the accuracy of the spectrum sensing may be affected. Finally, once the available spectrum holes are found, for characterizing each one, six decision parameters are calculated with the information contained into each decision matrix interval ID $_{n}$. In the following subsubsections, we describe how and why each of the decision parameters is calculated.
2.1.1. Duty Cycle $\left(\mathbf{d c}_{n}\right)$. A fundamental parameter to establish the spectral occupation from spectrum measurements is the duty cycle (DC). The DC is the percentage of time in which a signal power is above a threshold. Through the method of the energy detector, it is possible to know the DC for each frequency band (i.e., channel). It requires the energy threshold $\gamma$, which must be carefully selected to avoid estimation errors in the state of a channel. In the present work, the $\mathrm{m}-\mathrm{dB}$ criterion is used to set the decision threshold value [20]. Thus, we set the threshold value $10 \mathrm{dBm}$ over the noise floor according to the methodology suggestions made in $[20,21]$ and by the International Telecommunications Union (ITU) in [22]. Note that a high value of DC means that the PU is using this frequency band most of the time, and a low value of DC means that the PU is absent for a long period of time. Each $\mathrm{id}_{s, p}$ of $\mathbf{I D}_{n}$ is passed through $\gamma$, where 0 or 1 values are assigned on a $\mathbf{D C}$ matrix with same size as $\mathbf{I D}_{n}$, as explained by the following equation:

$$
\mathbf{D C}_{n}= \begin{cases}1, & \text { if } \mathbf{I D}_{n} \geq \gamma, \\ 0, & \text { if } \mathbf{I D}_{n}<\gamma .\end{cases}
$$

Thereafter, the $S$ rows of $\mathbf{D C} C_{n}$ are averaged. The result is stored in $\mathbf{d} \mathbf{c}_{n}$, as shown in (3). The $\mathbf{d} \mathbf{c}_{n}$ variable has the average DC for each frequency point $p$ with values between 0 and 1 for the decision $n$. Since a lower value of this parameter is the better, the DC represents a cost parameter.

$$
\mathbf{d c}_{n}=\frac{1}{S} \sum_{s=1}^{S} \mathbf{D C}_{n}, \quad[0,1] .
$$

2.1.2. Bandwidth $\left(\mathbf{b w}_{n}\right)$. One of the most important parameters to characterize spectrum holes is the bandwidth. Having a large value of bandwidth is preferable, since its size is related to the maximum data rate that can be attained by SU. Therefore, the bandwidth of a spectrum hole is considered a benefit parameter. Spectrum occupancy information can be utilized to determine the existence of spectrum holes. Here, using the information contained in $\mathbf{d} \mathbf{c}_{n}$ vector and the resolution points $p$, each spectrum hole is calculated. In this process, each element of $\mathbf{d c}_{n}$ vector is rounded in two values ( 0 or 1 ) in order to determine empty or occupied frequencies.

Algorithm 1 and Figure 2 describe how the $\mathbf{b w}_{n}$ vector is obtained. The beginning of a spectrum hole is considered when the $l$ counting variable is odd or equal to 0 and $\mathrm{dc}_{n}(p)<$ 1. In that case, the value of the auxiliary vector $\mathbf{f} \mathbf{v}_{n}$ to which $p$ is pointing out is stored in $f_{i}$ as can be seen in Figure 2. The spectrum hole ending appears when $\mathrm{dc}_{n}(p)=0$ and the next resolution point $\mathrm{dc}_{n}(p+1)>0$; in a similar way the $\mathbf{f} \mathbf{v}_{n}$ value where $p$ is pointing out is stored in $f_{f}$. Also, the size of this space is calculated by doing $f_{f}-f_{i}$; if it is larger than $\epsilon$, it is saved in $u_{n}$, wherein $r$ corresponds to all spectrum holes with $r=1, \ldots, R$. For each $r$, there is a identifier that stores the value of central frequency of each spectrum hole.

In our work presented in [13], only $6 \mathrm{MHz}, 7 \mathrm{MHz}$, and $8 \mathrm{MHz}$ spectrum holes were considered. However, due to the presence of several spectrum holes smaller than $6 \mathrm{MHz}$ (but larger than $5 \mathrm{MHz}$ ), spectrum holes of $5 \mathrm{MHz}$ are also 


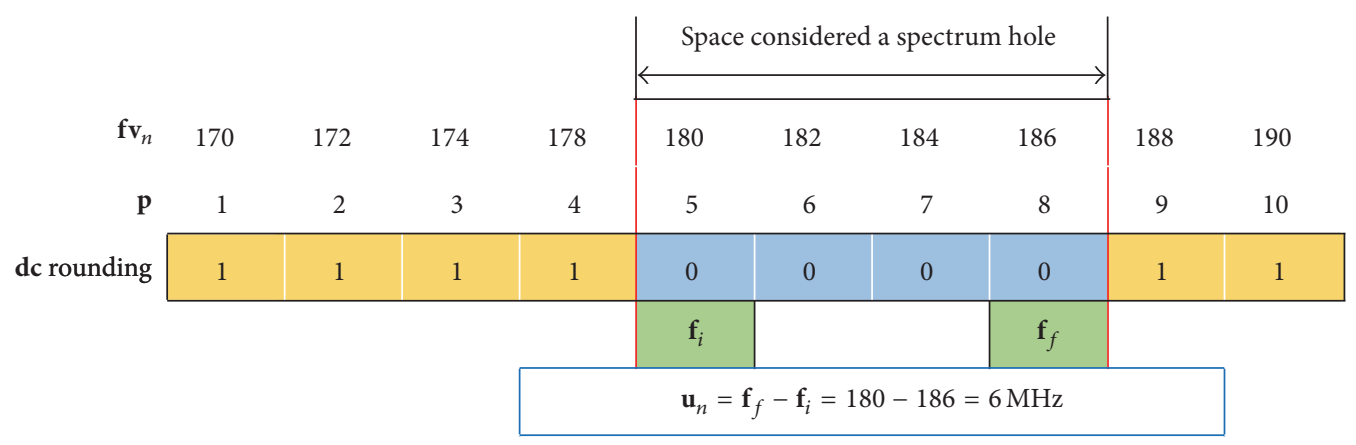

FIGURE 2: Example of how spectrum holes are found.

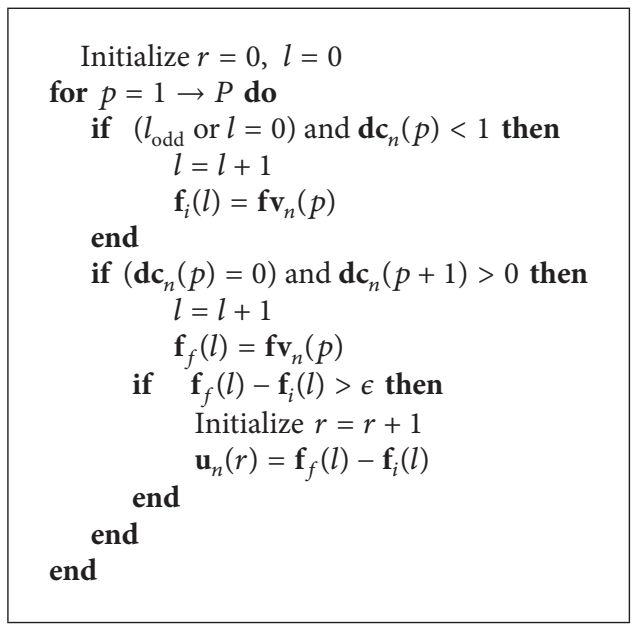

Algorithm 1: Bandwidth location.

considered in this work, and so $\epsilon=5 \mathrm{MHz}$. Contrary to IEEE 802.22 that does not consider spectrum holes smaller than $6 \mathrm{MHz}$, there are other wireless standards such as unlicensed LTE where this kind of empty spaces can be used [23, 24]. The four spectrum holes considered are the $a_{m}$ elements of the set $A=\left\{a_{1}=5, a_{2}=6, a_{3}=7, a_{4}=8\right\}$. If there is an element of $A$ equal to the spectrum hole size of $\mathbf{u}_{n}(r)$, it is directly assigned to $\mathbf{b} \mathbf{w}_{n}(k)$. In other cases, if the size of $\mathbf{u}_{n}(r)$ exceeds $8 \mathrm{MHz}$, it is randomly partitioned in the elements of $A^{\prime}$. The $A^{\prime}$ set includes random elements of $A$, as many as can be fitted in $A^{\prime}$. The condition is that the sum of $A^{\prime}$ elements should be less than or equal to $\mathbf{u}_{n}(r)$.

Finally, the vector with the average bandwidth size of each spectrum hole in the decision $n$ is $\mathbf{b w}_{n}$, where the $\mathbf{b w}_{n}$ length is $k=1, \ldots, K$, where $K$ is the number of spectrum holes per decision. It is important to mention that, similarly to $p$, the variable $k$ has an auxiliary vector $\mathbf{f k}_{n}$ where the central frequency values of each spectrum holes are stored. When $\mathbf{u}_{n}(r)$ is directly assigned to $\mathbf{b w}_{n}(k)$, the mapping $r$ to $k$ is one to one, for the contrary case $k$ will increase depending of the number of elements of $A^{\prime}$. Hence, if all spectrum holes of $\mathbf{u}_{n}$ are among the spectrum holes considered, $k=r$. However, when spectrum holes of $\mathbf{u}_{n}$ are larger, then $k>r$. Thus, the size of this vector is not fixed, and hence it can change for each spectrum decision.

2.1.3. Stability of Spectrum Holes $\left(\mathbf{s t}_{n}\right)$. Spectrum hole stability $\left(\mathbf{s t}_{n}\right)$ in time is a benefit parameter proposed in this work. A hole in the spectrum can appear to be always available, but that does not mean that it has the same size all the time. In fact, a spectrum hole can change in size affecting SU connection performance. Thus, this parameter measures how stable a spectrum hole is in time, and consequently the result provides the percentage of time that a spectrum hole is standing still.

In order to know the spectrum holes for each row $s$ of the $\mathbf{D C} C_{n}$ matrix, the vector $\mathbf{t h}_{s}$ is computed using the same procedures used for computing $\mathbf{b w}_{n}$. The result is stored in $\mathbf{t h}_{s}(g)$, where $g=1, \ldots, G$ with $G$ being the temporal variable with the number of spectrum holes found in that row $s$. The temporal variable $G$ has also an auxiliary vector $\mathbf{f g}_{s}$ where the central frequency values of each spectrum holes of $\mathbf{t h}_{s}$ are stored. After that, the $\mathbf{f} \mathbf{k}_{n}$ vector with the central frequency of each element of the $\mathbf{b w}_{n}$ vector is sought in the $\mathbf{f g}_{s}$ vector corresponding to the $\mathbf{t h}_{s}(g)$ vector. The procedure follows the following restrictions: a spectrum hole has a variation, but it is considered the same if $\mathbf{f g} \mathbf{g}_{s} \in \mathbf{f} \mathbf{k}_{n}$ and if the difference between $\mathbf{f k}_{n}(k)$ and the $\mathbf{f} \mathbf{g}_{s}(g)$ element is lower than $\mathscr{V}$ as is shown in (4) (the $\mathscr{V}$ variable is a threshold, and its value in this work is $1 \mathrm{MHz}$; the reason for this threshold value is explained in Section 5.1). In this case, the difference of change between $\mathbf{f g}_{s}(g)$ and $\mathbf{f k}_{n}(k)$ is calculated and the instability (i.e., variation in time of the spectrum hole) is posted to the $\mathbf{i e}_{s}(k)$ variable; in an opposite case, we assume that the spectrum hole has disappeared or has overly changed in size, and hence the instability is assumed and quantified as 1 also in $\mathbf{i e}_{s}(k)$, as can be seen in

$$
\mathbf{i e}_{s}(k)= \begin{cases}\left|\mathbf{f} \mathbf{k}_{n}(k)-\mathbf{f} \mathbf{g}_{s}(g)\right|, & \text { if } \mathbf{f} \mathbf{g}_{s}(g) \in \mathbf{f} \mathbf{k}_{n} \wedge \mathbf{f} \mathbf{k}_{n}(k)-\mathbf{f} \mathbf{g}_{s}(g)<\mathscr{V}, \\ 1, & \text { if } \mathbf{f} \mathbf{g}_{s}(g) \notin \mathbf{f} \mathbf{k}_{n} .\end{cases}
$$


The $\mathbf{i e}_{s}$ vector with values between 0 and 1 is stored in the $\mathbf{S T}_{n}$ matrix with dimension $S \times K$, as can be seen in

$$
\mathbf{S T}_{n}(s)=\mathbf{i e}_{s}, \quad[0,1]
$$

Finally, the rows of the matrix mentioned before are averaged, subtracted from 1 , and multiplied by 100 to obtain the percentage of stability $\mathbf{s t}_{n}$ for the decision $n$, as can be seen in

$$
\mathbf{s t}_{n}=\left(1-\frac{1}{S} \sum_{s=1}^{S} \mathbf{S} \mathbf{T}_{n}\right) * 100, \quad[0,100]
$$

2.1.4. Power Index ( $\left.\mathbf{p} \mathbf{i}_{n}\right)$. The power index is an important relation that should be considered in the spectrum decision process [13]. This relation indicates how a SU can be affected when a PU appears. A spectrum hole can be present for a long time, but if the PU requires using its frequency band, the PU can transmit suddenly and cause problems to the SU. This parameter quantifies how much a SU is affected by the phenomenon. For each frequency range that is considered a spectrum hole, (7) must be computed:

$$
\begin{array}{ll}
P_{\text {max }}(k)=\max _{s, p}\left(\mathbf{I D}_{n}\right), & \forall \mathbf{b} \mathbf{w}_{n}(k), \\
P_{\text {min }}(k)=\min _{s, p}\left(\mathbf{I D}_{n}\right), & \forall \mathbf{b} \mathbf{w}_{n}(k) .
\end{array}
$$

After that, the power index $\mathbf{p i}_{n}(k)$ for the decision $n$ is obtained by the division of $P_{\max }(k)$ by $P_{\min }(k)$, as can be seen in (8). If $P_{\max }(k)$ is close to $P_{\min }(k), \mathbf{p i}_{n}(k)$ is near 1 , and therefore the maximum power is low and the SU is not greatly affected by the PU presence. The $\mathbf{p i}_{n}(k)$ vector also can be near 1 when the minimum power is high; however, in that case the spectrum hole is occupied most of the time and it is not considered as a good candidate. This parameter is considered a benefit:

$$
\mathbf{p} \mathbf{i}_{n}(k)=\frac{P_{\max }(k)}{P_{\min }(k)}, \quad[0,1] .
$$

2.1.5. Interference Temperature (it ${ }_{n}$ ). A parameter that should be included in the selection of a spectrum hole is the interference temperature (IT). The frequency spectrum is saturated, and most of the available spectrum holes are surrounded by occupied frequencies; in this way, users on those frequencies can generate interference. This interference may cause deficiencies in performance of SUs. Thereby, in the selection process, knowing the corresponding IT of the spectrum holes is important. The concept of IT was initially proposed in [25]. In [26], two challenges to implement the ideal IT model are mentioned: the first one is identifying licensed signals, and the second one is measuring IT in the presence of the PU. For the second challenge, the author pointed out that PU knowledge facilitates IT determination. Thereby, calculating the approximation of IT as shown in [26] is possible by means of the following equation:

$$
\begin{aligned}
\mathbf{i t}_{n}(k) & \\
= & \frac{P\left(f_{c}-\mathbf{b w}_{n}(k) / 2-\tau\right)+P\left(f_{c}+\mathbf{b} \mathbf{w}_{n}(k) / 2+\tau\right)}{2 \mathscr{K} \mathbf{b} \mathbf{w}_{n}(k)},
\end{aligned}
$$

where $P$ is the power in the central frequency $f_{c}$ in the selected bandwidth $\mathbf{b w}_{n}(k), \mathscr{K}$ is Boltzmann's constant, and $\tau$ represents few $\mathrm{kHz}$ as guard band. The units of this parameter are Kelvin degrees and it is considered a cost parameter. In this parameter, a lower value of $\mathbf{i t}_{n}(k)$ is preferable in order to reduce interference and to obtain good communication performance. In (9), it has to be calculated for each spectrum hole in $\mathbf{b w}_{n}(k)$, where the $\mathbf{i t}_{n}$ vector is obtained for each decision $n$. The range of IT changes according to the frequency measured and the size of the available spectrum holes.

2.1.6. Selected Frequency $\left(\mathbf{s f}_{n}\right)$. The importance of frequency selection is based on the relation between the transmission frequency and the possible coverage zone of that transmission. Given an assigned value of power for performing a communication, here we assumed that the coverage zone for a lower frequency is expected to be larger than in a higher frequency. This means that choosing spectrum holes in lower frequency values is better than in upper frequency values. The communication process can be performed between farther points without increasing power transmission, which in turn may represent energy savings. However, it is worth mentioning that the considerations for this parameter may not be satisfactory for SUs near the SBSs and SUs at the edges of adjacent cells. To solve this issue, the interference has to be considered in the spectrum decision process and included as an additional decision parameter.

The spectrum holes that appear in $\mathbf{b w}_{n}$ receive a cost in the vector $\mathbf{s f}_{n}$. Equation (10) explains the distribution of penalty costs. The lowest possible frequency corresponds to the first spectrum hole $k=1$, which gets the lowest cost in $\mathbf{s f}_{n}$. The operation is repeated for each spectrum hole $K$ with the same cost in $\mathbf{s f}_{n}$ as the value of $K$; hence $\mathbf{s f}_{n}$ is the same size as $\mathbf{b} \mathbf{w}_{n}$ and it is calculated for each decision $n$. This parameter is a cost; a lower cost or a lower frequency is preferable instead of a high frequency:

$$
\begin{array}{ccc}
\mathbf{b w}_{n}(1) & \text { (lowest frequency); } & \mathbf{s f}_{n}(1)=1, \\
\vdots & \vdots & \vdots \\
\mathbf{b w}_{n}(K) & \text { (highest frequency); } & \mathbf{s f}_{n}(K)=K .
\end{array}
$$

\section{MADM-Based Spectrum Decision}

Multiple attribute decision-making (MADM) methods deal with decision problems where a selection has to be made over several available alternatives that are characterized each by multiple and usually conflicting attributes [4]. MADM is a branch of the field multiple criteria decision-making (MCDM). There are MADM-based decision problems in a diverse range of engineering disciplines, but they all share the following common characteristics: alternatives to select, multiple attributes describing the alternatives in different measurement units, and a set of weights representing the relative importance among attributes. In the following, the spectrum decision problem in CRNs is formulated as a MADM-based decision and the MADM methods considered in this work are presented. 
3.1. Decision Matrix $\left(\mathbf{D M}_{n}\right)$. First, according to the previous section, the available spectrum holes and their corresponding parameters are arranged as follows to form the decision matrix $\mathbf{D} \mathbf{M}_{n}$ for each decision $n$ as can be seen in

$$
\mathbf{D M}_{n}=\left(\begin{array}{cccccc}
\mathrm{bw}_{11} & \mathrm{st}_{12} & \mathrm{pi}_{13} & \mathrm{dc}_{14} & \mathrm{it}_{15} & \mathrm{sf}_{1 J} \\
\vdots & \vdots & \vdots & \vdots & \vdots & \vdots \\
\mathrm{bw}_{K 1} & \mathrm{st}_{K 2} & \mathrm{pi}_{K 3} & \mathrm{dc}_{K 4} & \mathrm{it}_{K 5} & \mathrm{sf}_{K J}
\end{array}\right) \text {, }
$$

where the first three columns correspond to benefit decision parameters, bandwidth bw, stability st, and power index pi, while the next three columns correspond to cost decision parameters, duty cycle dc, interference temperature it, and selected frequency sf. Thus, in this paper $J=6$ always in (11). On the other hand, the $K$ rows correspond to the $K$ available spectrum holes at decision $n$, and thus $K$ may vary from decision to decision based on the occupancy of the spectrum. Note that in (3) dc was in terms of $p$; however, for the $\mathbf{D M}_{n}$ matrix $\mathbf{d c}$ is resized with the corresponding duty cycle of the spectrum holes found.

It is important to mention that although the spectrum decision MADM-based formulation presented in this work considers only decision parameters extracted from the spectrum measurements (i.e., physical layer level), other types of parameters may be considered. For example, parameters describing the network-level performance of the network may be considered such as packet delay and jitter, security level, and access cots. In fact, network-level parameters must be considered for spectrum decision in scenarios with several coexisting CRNs. Nevertheless, such scenario can be also considered with the presented MADM-based approach by extending the number of columns to the decision matrix $\mathbf{D M}_{n}$.

3.2. Connection Profiles. As mentioned before, a set of connection profiles are assumed to be prestored in the SU terminal. Once the decision matrix $\mathbf{D M}_{n}$ is defined, it is required to assign the different level of importance or relevance of each decision parameter. This level of importance is established in the connection profile which is defined by the set of the corresponding six importance weights. The sum of those weights has to satisfy the constraint $\sum_{j=1}^{J} w_{j}=1$ where $w_{j}$ corresponds to the importance weight of parameter $j$ with $J=6$. From the six decision parameters defined in Section 2.1 and considered in the proposed spectrum decision formulation, the bandwidth size of the spectrum hole bw is the one with major impact in the connection requirements since it is directly related to the maximum achievable data rate among the SU terminal and the SBS. Thus, here such parameter will be assumed to be the one ensuring the connection requirements. However, in order to have a more accurate connection profile, network-level parameters should be considered as discussed before.

The $\mathbf{D} \mathbf{M}_{n}$ matrix and the set of importance weights $w_{j}$ are the input information to the MADM algorithms. The MADM algorithms first calculate a score for each alternative based on their specific procedures, and then the alternative with the best value score is selected. Usually, the alternatives are listed in ranking, and thus the first place in the ranking list is the selected option. Hence, the final result of each MADM algorithm is the selection of a row (i.e., a spectrum hole) from $\mathbf{D M}_{n}$. To make the notation easier, for each decision $n$, this row is given by selected spectrum hole $\mathbf{h s}_{n}$. It includes the six decision parameters, as can be seen in

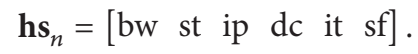

In the following subsections, the MADM algorithms implemented in this work are presented.

3.3. Simple Additive Weighting (SAW). This algorithm was introduced for decision process in wireless networking in [27]; SAW is also called the weighted sum method. The variable at ${ }_{k j}$ corresponds to the normalization of each decision parameter and $\mathrm{dm}_{k j}$ is an element of the $\mathbf{D} \mathbf{M}_{n}$ matrix. This method performs homogenization; each at ${ }_{k j}$ parameter is multiplied by $w_{j}$ to obtain the score of each candidate alternative. The normalization of $\mathrm{at}_{k j}$ is described as follows: if at $_{k j}$ is a benefit parameter, it is normalized as can be seen in (13), where $\mathrm{dm}_{j}^{+}=\max _{k} \mathrm{dm}_{k j}$. On the other hand, if at $\mathrm{t}_{k j}$ is a cost parameter, it is normalized as it is shown in (14), where $\mathrm{dm}_{j}^{-}=\min _{k} \mathrm{dm}_{k j}$ :

$$
\begin{aligned}
& \mathrm{at}_{k j}=\frac{\mathrm{dm}_{k j}}{\mathrm{dm}_{j}^{+}}, \\
& \mathrm{at}_{k j}=\frac{\mathrm{dm}_{j}^{-}}{\mathrm{dm}_{k j}} .
\end{aligned}
$$

The alternative selected by this method is (15), where $w_{j}$ is the importance weight of $j$ th parameter:

$$
\mathbf{h s}_{\mathrm{SAW}}^{*}=\arg \max _{k} \sum_{j=1}^{J} w_{j} \mathrm{at}_{k j} \text {. }
$$

3.4. Technique for Order Preference by Similarity to Ideal Solution (TOPSIS). TOPSIS is another MADM method, which was also used in wireless networking in [27]. This algorithm calculates the alternative with the shortest Euclidian distance being the ideal alternative and the alternative with the largest Euclidian distance to the negative-ideal solution, using the information contained in $w_{j}$ and $\mathrm{dm}_{i j}$. TOPSIS performs the following steps in order to compute the alternatives ranking list.

Step 1. Construct the normalized decision matrix, which allows comparison across the attributes as

$$
\mathrm{at}_{k j}=\frac{\mathrm{dm}_{k j}}{\sqrt{\sum_{k=1}^{K} \mathrm{dm}_{k j}^{2}}} .
$$

Step 2. Construct the weighted normalized decision matrix as $v_{k j}=w_{j} * \mathrm{at}_{K j}$. 
Step 3. Determine ideal alternative with (17) and negativeideal alternatives with (18) as follows:

$$
\begin{aligned}
& \mathrm{Al}^{+}=\left\{\left(\max _{k} v_{k j} \mid j \in \mathrm{BC}\right),\left(\min _{k} v_{k j} \mid j \in \mathrm{BC}^{\prime}\right)\right\}, \\
& \mathrm{Al}^{-}=\left\{\left(\min _{k} v_{k j} \mid j \in \mathrm{BC}\right),\left(\max _{k} v_{k j} \mid j \in \mathrm{BC}^{\prime}\right)\right\},
\end{aligned}
$$

where $\mathrm{BC}$ is the set of benefit parameters and $\mathrm{BC}^{\prime}$ is the set of cost parameters.

Step 4. Calculate the distance between the ideal positive alternatives with (19) and the negative-ideal alternatives with (20) as follows:

$$
\begin{gathered}
s_{k}^{+}=\sqrt{\sum_{j=1}^{J}\left(v_{k j}-v_{j}^{+}\right)^{2}}, \\
s_{k}^{-}=\sqrt{\sum_{j=1}^{J}\left(v_{k j}-v_{j}^{-}\right)^{2}} .
\end{gathered}
$$

Step 5. Calculate the relative closeness to the ideal alternative:

$$
c_{k}^{*}=\frac{s_{k}^{-}}{\left(s_{k}^{+}+s_{k}^{-}\right)} .
$$

Equation (22) corresponds to the final step of this algorithm, where the best spectrum hole according to the SU's connection requirements is $\mathbf{h s}_{\mathrm{TOP}}^{*}$ :

$$
\mathbf{h s}_{\mathrm{TOP}}^{*}=\arg \max _{k} c_{k}^{*} \text {. }
$$

3.5. VIKOR. The VIKOR compromise ranking method was proposed for wireless networks in [28]. This method selects an alternative close to the ideal among the rest of the alternatives. This algorithm carries out the following steps.

Step 1. For each parameter $j=1,2,3, \ldots, J$, determine the best value (23) and the worst value (24) given by

$$
\begin{aligned}
& F_{j}^{+}=\left\{\left(\max _{k} \mathrm{dm}_{k j} \mid j \in J_{b}\right),\left(\min _{k} \operatorname{dm}_{k j} \mid j \in J_{c}\right)\right\}, \\
& F_{j}^{-}=\left\{\left(\min _{k} \mathrm{dm}_{k j} \mid j \in J_{b}\right),\left(\max _{k} \operatorname{dm}_{k j} \mid j \in J_{c}\right)\right\},
\end{aligned}
$$

where $J_{b} \subset \mathrm{BC}$ is the set of benefit parameters and $J_{c} \subset \mathrm{BC}^{\prime}$ is the set of cost parameters.

Step 2. Compute the values of $S_{k}(25)$ and $R_{k}(26)$ with $k=1$, $2,3, \ldots, K$ :

$$
\begin{aligned}
S_{k} & =\sum_{j=1}^{J} w_{j} \frac{\left(F_{j}^{+}-\mathrm{dm}_{k j}\right)}{\left(F_{j}^{+}-F_{j}^{-}\right)}, \\
R_{k} & =\max _{j}\left[w_{j} \frac{\left(F_{j}^{+}-\mathrm{dm}_{k j}\right)}{\left(F_{j}^{+}-F_{j}^{-}\right)}\right],
\end{aligned}
$$

where $w_{j}$ is the importance weight of parameter $j$.
Step 3. Compute the values of $Q_{k}$ with (27) for $k=1,2$, $3, \ldots, K$, where $\gamma$ is the weight of the strategy with $0 \leq \gamma \leq 1$ :

$$
Q_{k}=\gamma\left(\frac{S_{k}-S^{+}}{S^{-}-S^{+}}\right)+(1-\gamma)\left(\frac{R_{k}-R^{+}}{R^{-}-R^{+}}\right),
$$

where

$$
\begin{aligned}
& S^{+}=\min _{k} S_{k}, \\
& S^{-}=\max _{k} S_{k}, \\
& R^{+}=\min _{k} R_{k}, \\
& R^{-}=\max _{k} R_{K} .
\end{aligned}
$$

Step 4. Given the values of $Q$ for all $k \in K$, rank the candidate networks in an increasing order.

Equation (29) expresses the final step of this algorithm in the $\mathbf{h s}_{\mathrm{VIK}}^{*}$ vector, where $Q_{k}^{*}$ is the best solution for satisfying the SU's connection requirements.

$$
\mathbf{h s}_{\mathrm{VIK}}^{*}=\arg \min _{k} Q_{k}^{*} .
$$

\section{Comparison Function of the EMSD}

This section presents the Comparison Function of the EMSD that performs the tradeoff among handoff execution and its related energy consumption. Basically, the result of this function depends on the selected spectrum hole characteristics in the time before $\mathbf{h s}_{n-1}$, the selected spectrum hole in the current time $\mathbf{h} \mathbf{s}_{n}$, and ECCS. This function compares and decides the best spectrum hole that satisfies SU's connection requirements, while trading off energy consumption. In order to set units in the same range and compare the results, normalization is required [8].

4.1. Energy Consumption due to Channel Switching $\left(E_{c s_{n}}\right)$. An important issue in wireless communication technologies is energy consumption, which can be reduced through less complex algorithms, among several solutions [3, 4]. In CRNs, however, additional issues related to energy consumption should be considered since CR devices necessitates additional tasks. In [5], an energy consumption model is presented where the authors consider energy consumption due to transmission $\left(E_{\mathrm{tx}}\right)$, circuits $\left(E_{c}\right)$, channel switching $\left(E_{\mathrm{cs}}\right)$, and idling $\left(E_{d}\right)$. This paper is focused on calculating the $E_{\mathrm{cs}}$, given that this parameter is related to the execution of spectrum handoffs due to energy consumed by the RF front-end in the reconfiguration process to use a new transmission frequency. According to $[5,29-31], E_{\mathrm{cs}}$ can be obtained by the power dissipation for switching $P_{c}$ and channel switching latency $T_{c}$. To adapt $T_{c}$ to our notation, it is calculated as follows:

$$
T_{c}=t_{\mathrm{cs}}\left|f_{\mathrm{hs}_{n-1}}-f_{\mathrm{hs}_{n}}\right|
$$

where $t_{\mathrm{cs}}$ is the time delay for switching multiplied by the difference of the central frequency of the previously selected 
spectrum hole $f_{\mathrm{hs}}$ minus the central frequency of the new selected spectrum hole $f_{\mathrm{hs}_{n}}$, as shown in

$$
E_{\mathrm{cs}_{n}}=P_{\mathrm{cs}} T_{\mathrm{cs}}=P_{\mathrm{cs}}\left(t_{\mathrm{cs}}\left|f_{\mathrm{hs}_{n-1}}-f_{\mathrm{hs}_{n}}\right|\right) .
$$

4.2. Comparison Function Description. In the system model presented in Figure 1, multiple spectrum decisions are performed continuously; the proposed Comparison Function is executed after the preliminary decision of the MADM algorithms but before the final decision returned to the SU via SBS. The preliminary selection offered by MADM algorithms in CRNs presents an issue. In our work in [13], we demonstrated how these algorithms satisfy SUs connection profiles and provide the best spectrum hole selections. However, the MADM algorithms do not take into account additional expenses that a SU can have in order to reach the suggestion made for them, showing that they need to be improved for CRNs. For example, let us suppose that a SU is occupying a spectrum hole $\mathbf{h} \mathbf{s}_{n-1}$; after a certain time the next spectrum decision has to be executed. In the moment of the decision, there is a spectrum hole $\mathbf{h} \mathbf{s}_{n}$ similar to $\mathbf{h} \mathbf{s}_{n-1}$ but with a minor improvement (e.g., in bandwidth) than the actual one occupied by the SU; however, this spectrum hole is located in a very distant frequency band. The MADM algorithms are going to recommend to $S \mathrm{U} \mathbf{h} \mathbf{s}_{n}$ no matter what the necessary resources to reach it are. Therefore, the SU will waste a considerable amount of ECCS $E_{\mathrm{cs}}$ in comparison with the marginal improvement of switching to $\mathbf{h} \mathbf{s}_{n}$. In this stage, to enhance the MADM algorithms performance, a balance between $E_{\mathrm{cs}}$ and spectrum holes characteristics is required.

The Comparison Function of EMSD is an adapter to improve the MADM algorithms performance in CRNs. Thus, the Comparison Function set the equivalence between the parameters of the spectrum holes and the energy consumed by switching among frequencies. However, spectrum holes parameters and $E_{\mathrm{cs}}$ are in different units; nevertheless they can be compared after a normalization process [8]. Here, it is important to emphasize that the Comparison Function is dependent of the MADM algorithms and works as a complement. Also, it is important to point out that if $\mathbf{h} \mathbf{s}_{n-1}$ is no longer available, the Comparison Function is not used and only the decision of MADM algorithms is taken into account. The whole process is carried out under certain restrictions that are explained below.

This Comparison Function starts for $n>1$. The selected spectrum hole in a previous time $\mathbf{h s}_{n-1}$, the selected spectrum hole in the present time $\mathbf{h} \mathbf{s}_{n}$, and the average spectrum holes in the current time $\mathbf{D M}_{n}$ are necessary. The algorithm has two restrictions. The first one is $\mathbf{h} \mathbf{s}_{n} \neq \mathbf{h} \mathbf{s}_{n-1}$; that is, the selected spectrum holes must be different, whereas an opposite case means the selection has no changes and the comparison is not necessary. The second one is $\mathbf{h} \mathbf{s}_{n-1} \in \mathbf{D M}_{n}$; that is, the selected spectrum hole in the previous time $\mathbf{h s}_{n-1}$ should be included in $\mathbf{D M}_{n}$ in the present time, and this is why if $\mathbf{h} \mathbf{s}_{n-1}$ is not present in the current time, it has disappeared, which means that it is not possible to perform the comparison and the best selection is $\mathbf{h s}_{n}$.
The Comparison Function calculates two important values. The first calculation corresponds to the $E_{\mathrm{cs}_{n}}^{\prime}$, as can be seen in (32). Here, the unit-based normalization was implemented. The $\max \left(E_{\mathrm{cs}}\right)$ and the $\min \left(E_{\mathrm{cs}}\right)$ values depend on the frequency range and spectrum holes size considered:

$$
E_{\mathrm{cs}_{n}}^{\prime}=\frac{E_{\mathrm{cs}_{n}}-\min \left(E_{\mathrm{cs}}\right)}{\max \left(E_{\mathrm{cs}}\right)-\min \left(E_{c s}\right)}, \quad[0,1] .
$$

Also, the Comparison Function computes the changes among parameters of the selected spectrum holes. Every selected spectrum hole has six decision parameters, as shown in (12). The algorithm measures the distance between the parameters of $\mathbf{h} \mathbf{s}_{n}$ and the parameters of $\mathbf{h} \mathbf{s}_{n-1}$. Thus, every element of $\mathbf{h} s_{n}$ is subtracted from every element of $\mathbf{h} \mathbf{s}_{n-1}$; the subtraction is made between parameters of the same kind. After that, each parameter subtraction uses the absolute value and it is normalized following the max-min method $[8,32]$. This procedure is done in order to get a proportion of all decision parameters, which define the properties of each spectrum hole. For example, for a benefit parameter such as $\mathrm{bw}$, the normalization process is made according to

$$
\mathrm{bw}^{\prime}=\frac{\left|\mathrm{bw}_{\mathrm{hs}_{n}}-\mathrm{bw}_{\mathrm{hs}_{n-1}}\right|}{\max (\mathrm{bw})-\min (\mathrm{bw})}, \quad[0,1] .
$$

On the other hand, for a cost parameter such as dc, the process is made according to

$$
\mathrm{dc}^{\prime}=\frac{\max (\mathrm{dc})-\min (\mathrm{dc})}{\left|\mathrm{dc}_{\mathrm{hs}_{n}}-\mathrm{dc}_{\mathrm{hs}_{n-1}}\right|}, \quad[0,1] .
$$

Thereby, the resultant vector with the proportion of all parameters $\mathbf{r}_{n}$ is conformed as shown in

$$
\mathbf{r}_{n}=\left[\begin{array}{llllll}
\mathrm{bw}^{\prime} & \mathrm{st}^{\prime} & \mathrm{pi}^{\prime} & \mathrm{dc}^{\prime} & \mathrm{it}^{\prime} & \mathrm{sf}^{\prime}
\end{array}\right] .
$$

In order to quantify $\mathbf{r}_{n}$ in a scalar number that can be compared with $E_{\mathrm{cs}_{n}}^{\prime}$, the values of $\mathbf{r}_{n}$ are summed. Finally the result is normalized again in $r_{n}^{\prime}$ as can be seen in

$$
r_{n}^{\prime}=\frac{1}{J} \sum_{j=1}^{J} \mathbf{r}_{n_{j}}, \quad[0,1]
$$

After that, as $E_{\mathrm{cs}_{n}}$ and $r_{n}$ are under the same range, it is possible to compare them. The algorithm looks for the minimum value between $r_{n}^{\prime}$ and $E_{\mathrm{cs}_{n}}^{\prime}$. The result is assigned to $d_{n}$ as

$$
d_{n}=\min \left(E_{\mathrm{cs}_{n}}^{\prime}, r_{n}^{\prime}\right) .
$$

Afterwards, the final selected spectrum hole $\mathbf{f} \mathbf{h}_{n}$ for the decision $n$ is obtained with (38). If $d_{n}$ is equal to $E_{\mathrm{cs}_{n}}^{\prime}$, which means that the ECCS does not involve excessive cost and the benefits offered by the new selected spectrum hole $\mathbf{h} \mathbf{s}_{n}$ are better than the current one, hence the device should take $\mathbf{h s}_{n}$. The contrary case points out that it is better to keep the 


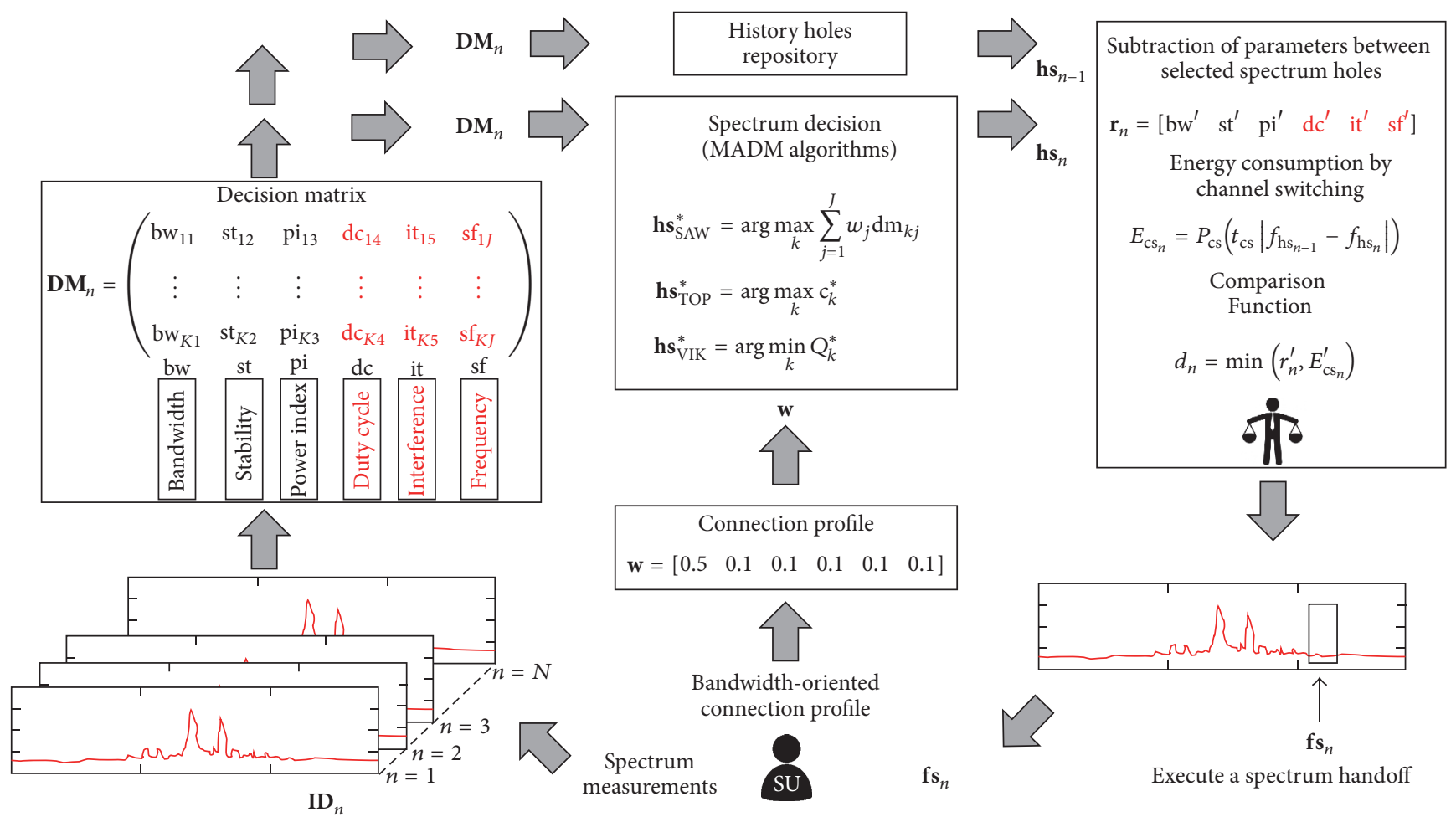

FIGURE 3: Summary of the EMSD, a spectrum decision proposal for an SU bandwidth-oriented connection profile.

previously selected spectrum hole $\mathbf{h s}_{n-1}$, because the benefits of $\mathbf{h s}_{n}$ are lower than the $E_{\mathrm{cs}_{n}}$ :

$$
\mathbf{f h}_{n}= \begin{cases}\mathbf{h} \mathbf{s}_{n}, & \text { if } d_{n}=E_{\mathrm{cs}_{n}}^{\prime} \\ \mathbf{h} \mathbf{s}_{n-1}, & \text { if } d_{n}=r_{n}^{\prime}\end{cases}
$$

Thus, $\mathbf{f h}_{n}$ is the result of this Comparison Function, which can be integrated to any MADM algorithm.

4.3. EMSD Summary. In this section, we explain an example of how our EMSD proposal works. Figure 3 summarizes all the parts included in this proposal. As noted above, the proposal runs after the first decision $(n=2)$. In the ID $_{2}$ matrix, the spectrum measurements between spectrum decisions $n=1$ and $n=2$ are considered. All six decision parameters presented in the previous section are extracted form that matrix. These parameters comprise the decision $\mathbf{D M}_{2}$ matrix, which is stored and at the same time sent to the MADM algorithms. For this example, we consider a SU with a bandwidth-oriented connection profile. Thus, w has the highest weight in the bandwidth parameter. MADM algorithms select the best spectrum hole according to the connection profile and the spectrum holes in $\mathbf{D} \mathbf{M}_{2}$. The selected spectrum hole $\mathbf{h s}_{2}$ is compared with the previous one $\mathbf{h s}_{1}$, which was stored in the history holes repository. The Comparison Function evaluates whether it is suitable to change to $\mathbf{h s}_{2}$ or remain in $\mathbf{h s}_{1}$, taking into account each spectrum hole characteristic and the ECCS. Finally, according to its connection profile, the final selected spectrum hole $\mathbf{f s}_{n}$ is delivered to the SU to continue or not with the execution of the spectrum handoff.

\section{Results}

This section presents a performance comparison among the MADM algorithms (SAW, TOPSIS, and VIKOR) standalone and the same MADM algorithms when utilizing the Comparison Function of our EMSD proposal. Also, as a reference a random spectrum hole selection is included in the results. To evaluate the performance, the algorithms are executed over real spectrum holes from a spectrum occupancy measurements campaign described below. To achieve high accuracy results, this spectrum decision process is simulated by means of a 1000-round Montecarlo method using MATLAB. The tables and plots correspond to the average values of the selected spectrum holes. Also, the results are quantified in terms of number of spectrum handoffs and ECCS per each algorithm. In this paper, two connection profiles are considered. The first one is similar weights-oriented profile; it occurs when a SU does not have a specific preference for any parameter in the spectrum hole. The second connection profile is the bandwidth-oriented profile, when a SU has a preference on getting a spectrum hole with the largest value of bandwidth.

5.1. Spectrum Occupancy Measurements on TV Bands. Realistic results can be obtained with information coming from real data. In [14], the spectrum opportunities for CRNs below $1 \mathrm{GHz}$ were shown. In this work, data from a spectrum occupancy campaign are included. We focused the 
TABLE 1: Average selected spectrum holes for a similar weights-oriented connection profile.

\begin{tabular}{|c|c|c|c|c|c|c|}
\hline Algorithm & bw & st & pi & dc & it & sf \\
\hline SAW & 6.2 & 66.8 & 0.98 & $6.74 * 10^{-5}$ & $3.59 * 10^{4}$ & 509.9 \\
\hline SAW + Comparison Function & 5.8 & 77.1 & 0.97 & $3.73 * 10^{-4}$ & $1.66 * 10^{5}$ & 406.7 \\
\hline TOPSIS & 6.2 & 76.1 & 0.97 & $2.86 * 10^{-4}$ & $2.67 * 10^{4}$ & 587.2 \\
\hline TOPSIS + Comparison Function & 6.1 & 71.6 & 0.97 & $2.66 * 10^{-4}$ & $2.73 * 10^{4}$ & 581.2 \\
\hline VIKOR & 6.1 & 60.4 & 0.98 & $6.15 * 10^{-4}$ & $1.18 * 10^{5}$ & 463.6 \\
\hline VIKOR + Comparison Function & 5.4 & 87.9 & 0.97 & 0.001 & $3.52 * 10^{5}$ & 252.5 \\
\hline Random & 6.1 & 50.9 & 0.97 & 0.009 & $2.06 * 10^{5}$ & 449.5 \\
\hline
\end{tabular}

spectrum measurements on frequencies with low DC in TV frequency bands. The proposed methodology for this campaign includes three observation points, which were set to detect the spectrum usage surrounding a specific zone within the city. This study considered the frequencies from $170 \mathrm{MHz}$ to $220 \mathrm{MHz}, 525 \mathrm{MHz}$ to $575 \mathrm{MHz}$, and $625 \mathrm{MHz}$ to $675 \mathrm{MHz}$ in the Mexican city of San Luis Potosí. Similar equipment used in [14] was used to obtain the spectrum usage information. The results showed a low presence of PUs with an average DC of $20 \%$. Spectrum measurements were taken in each observation point around $24 \mathrm{hrs}$ for each frequency range in a week day. For each observation point, the spectrum samples were averaged according to each frequency range. Thus, for each frequency range an average of 80000 spectrum usage samples were obtained; these samples were used for each Montecarlo round. In order to process this information, $N=80$ spectrum decisions were made. The samples of each spectrum decision were assigned into the matrix ID $_{n}$. Thus, a spectrum decision was conducted each $1.25 \%$ of the total spectrum time analyzed, where $S=1000$ samples and the number of resolution points of the frequencies examined is $P=1500$. Given that, the spectrum measurements come from TV frequency bands, and we considered the technical details from the IEEE 802.22 standard [33]. This guideline points out that there is an effective signal bandwidth of $5.5 \mathrm{MHz}$ for a $6 \mathrm{MHz} \mathrm{TV}$ channel. Thus, the remaining $0.5 \mathrm{MHz}$ is twice the size of the value of $\mathscr{V}$ mentioned in Section 2.1.3, and the value of $\tau$ presented in Section 2.1.5 is once that remaining value. For the ECCS presented in Section 4.2, we have assigned the values taken in [5]: $P_{\mathrm{cs}}=$ $1000 \mathrm{~mW}$ and $t_{\mathrm{cs}}=0.1 \mathrm{~ms} / \mathrm{MHz}$, and thus $E_{\mathrm{cs}}$ is given in Joules.

5.2. Similar Weights-Oriented Connection Profile. In this case, the SU connection requirements do not have a preference over any of the decision parameters; the vector of importance weights $\mathbf{w}$ receives equal values. Thus, this connection profile requires an available spectrum hole with affordable characteristics in all parameters. The average values of the six parameters corresponding to the selected spectrum holes appear in Table 1 . Note that all the selected spectrum holes have an average bandwidth size of approximately $6 \mathrm{MHz}$. However, by combining MADM algorithms and the Comparison Function, now spectrum holes in lower frequencies are selected. This might represent a larger coverage zone and energy savings for the SU. For example, by using VIKOR combined with the function, the SU obtains on average spectrum holes with $0.7 \mathrm{MHz}$ less bandwidth compared by
VIKOR alone. On the contrary, in this same example, the average of the selected spectrum holes exhibits the lowest selected frequency in $252 \mathrm{MHz}$ and also the stability increases $27 \%$ compared to VIKOR alone. The random selection obtains average values in all the parameters, except for the lowest stability with $51 \%$.

Figure 4(a) shows a significant reduction in the number of spectrum handoffs when the Comparison Function is used. For the considered cases, the number of spectrum handoffs successfully avoided is approximately from 20 to 60. It can be seen that MADM algorithms (alone) reduce between 5 and 30 the number of spectrum handoffs when compared to the random selection. Moreover, the combination MADM algorithms with Comparison Function obtain between 50 and 60 less spectrum handoffs compared to the random case. As expected, this reduction is directly related to a decrement in the amount of ECCS as shown in Figure 4(b). The ECCS saved by using the Comparison Function for SAW and TOPSIS is approximately of 4 Joules. Nevertheless, combining VIKOR plus the Comparison Function saves on average about 15 Joules. However, the combination of MADM algorithms with Comparison Function saves between 18 and 19 Joules compared to the random selection. Thus, the inconvenient ping-pong effect due to frequent spectrum handoffs is avoided and the energy saved at the SUs battery is available for other tasks.

5.3. Bandwidth-Oriented Connection Profile. For this case, the SU connection requirements demand a large amount of bandwidth. Thus, the bandwidth parameter has the highest weight, with a 1:5 proportion with respect to the other decision parameters. The decision algorithms look for the spectrum hole with the largest bandwidth and give less importance to the other parameters. But still the MADM algorithms have to consider the six parameters in the spectrum decision. Table 2 shows the average values of the selected spectrum holes for this connection profile. In this table, the number of selected spectrum holes increased the bandwidth size with respect to the previous connection profile. Note that VIKOR and the combination of VIKOR with Comparison Function select on average the largest values of bandwidth. Also the combination of VIKOR plus Comparison Function achieves on average the lowest selected frequency with $292.9 \mathrm{MHz}$. On the other hand, SAW and TOPSIS (both alone) present similar results in bandwidth size, power index, and interference temperature. However, when SAW and TOPSIS include the Comparison Function, 


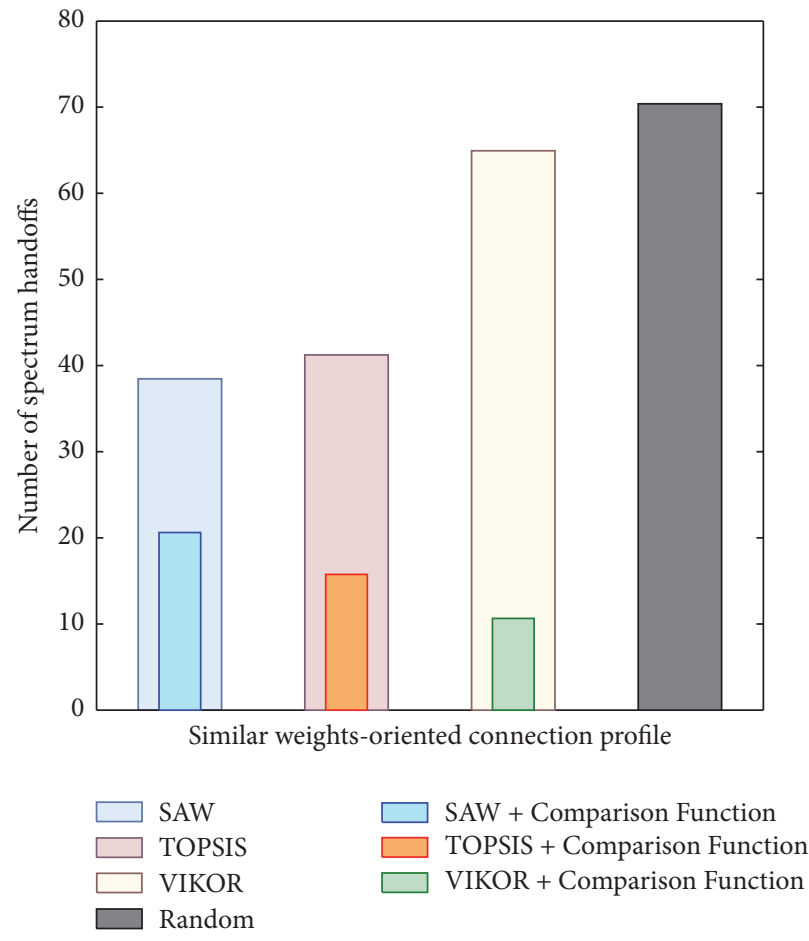

(a) Number of spectrum handoffs

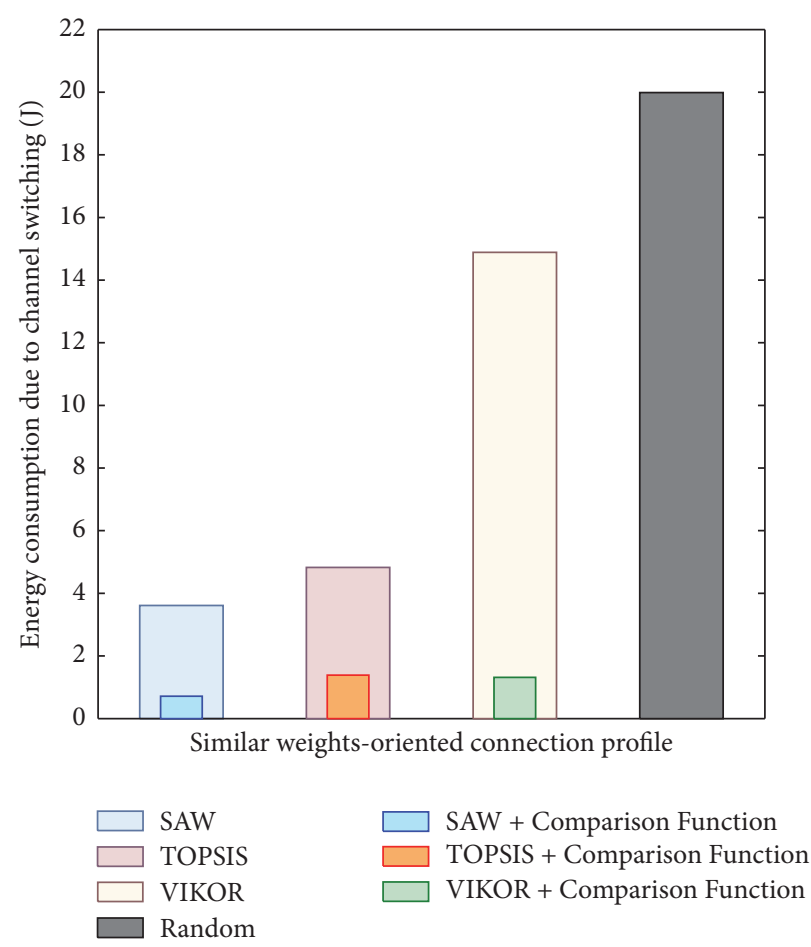

(b) Energy consumption due to channel switching

FIGURE 4: Average performance of MADM algorithms, MADM algorithms combined with Comparison Function of the EMSD and a random case for a similar weights-oriented connection profile.

TABLE 2: Average selected spectrum holes for a bandwidth-oriented connection profile.

\begin{tabular}{|c|c|c|c|c|c|c|}
\hline Algorithm & bw & st & pi & dc & it & sf \\
\hline SAW & 6.6 & 63.5 & 0.98 & $5.53 * 10^{-4}$ & $2.91 * 10^{4}$ & 529.2 \\
\hline SAW + Comparison Function & 6.2 & 69.1 & 0.97 & 0.003 & $1.58 * 10^{5}$ & 448.8 \\
\hline TOPSIS & 6.6 & 67.1 & 0.98 & $3.46 * 10^{-4}$ & $2.49 * 10^{4}$ & 584.8 \\
\hline TOPSIS + Comparison Function & 6.3 & 68.5 & 0.98 & $3.45 * 10^{-4}$ & $3.59 * 10^{4}$ & 573.4 \\
\hline VIKOR & 6.8 & 35.8 & 0.97 & 0.022 & $3.11 * 10^{5}$ & 427.7 \\
\hline VIKOR + Comparison Function & 6.8 & 25.4 & 0.97 & 0.039 & $5.61 * 10^{5}$ & 292.9 \\
\hline Random & 6.1 & 50.7 & 0.97 & 0.009 & $2.05 * 10^{5}$ & 449.9 \\
\hline
\end{tabular}

they present a reduction of $0.3 \mathrm{MHz}$ in the average bandwidth size. Nevertheless, the stability increases $2-6 \%$ more in both algorithms while for SAW plus the Comparison Function a reduction of the selected frequency to $448.8 \mathrm{MHz}$ is attained as well. Finally, the random selection as expected achieves similar values to the previous user profile.

Figure 5(a) shows an increment of 8-10 more spectrum handoffs only for SAW and TOPSIS compared to the similar weights-oriented profile. However, for this connection profile the number of spectrum handoffs avoided is approximately from 20 to 55. The combination of MADM algorithms plus the Comparison Function compared to the random selection avoids between 45 and 55 spectrum handoffs. MADM algorithms (alone) obtain between 8 to 21 spectrum handoffs less than random case. Similarly to the previous results, an augmentation in the number of spectrum handoffs generates an increment in the amount of ECCS as shown in Figure 5(b). The energy saved by the Comparison Function follows the trend of the last connection profile, 2-15 Joules. An important amount of ECCS is saved by the MADM plus the Comparison Function compared to the random selection. The first algorithm consumes between 2 and 3 Joules, while the random case consumes 20 Joules. Therefore, it is clearly shown that the use of the Comparison Function always reduces more than half of the energy consumed in all cases.

Figure 6 shows the average value of bandwidth as well as the selected frequency of the selected spectrum holes during the spectrum decision process of the 80 successive decisions by SAW and SAW combined with the Comparison Function for a bandwidth-oriented connection profile. From this figure, it is easy to see how the EMSD proposal avoids an important number of spectrum handoffs. Note also in the same figure that SAW combined with the Comparison Function tends to select spectrum holes in lower values of frequencies. On the other hand, both options select spectrum holes with similar bandwidth sizes. However, the SU which 


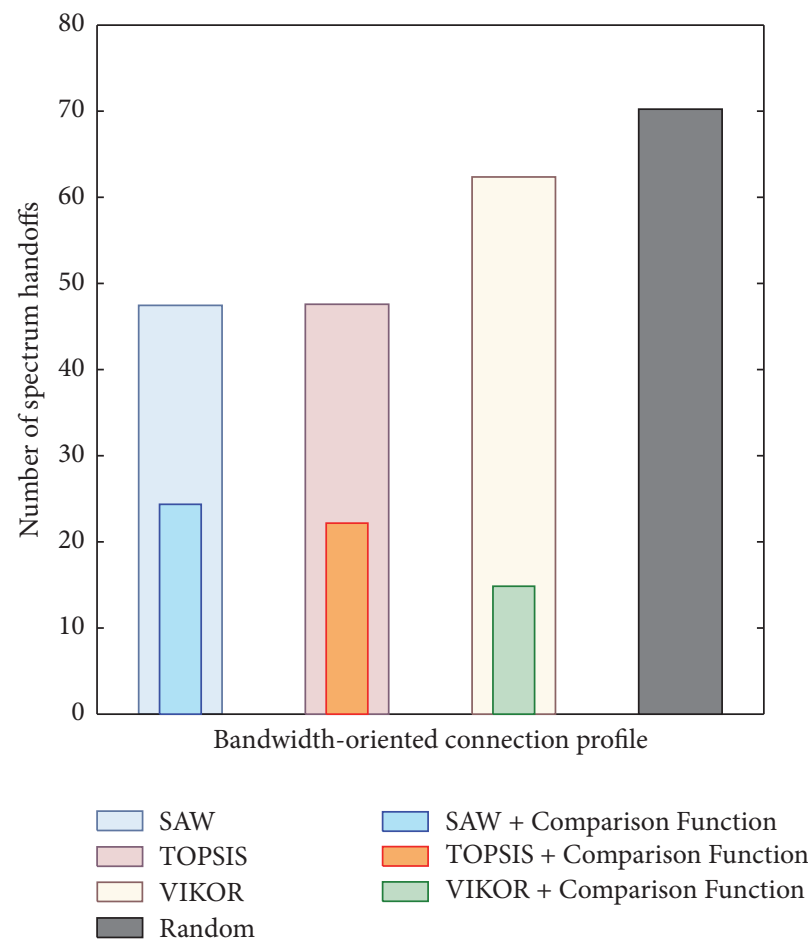

(a) Number of spectrum handoffs

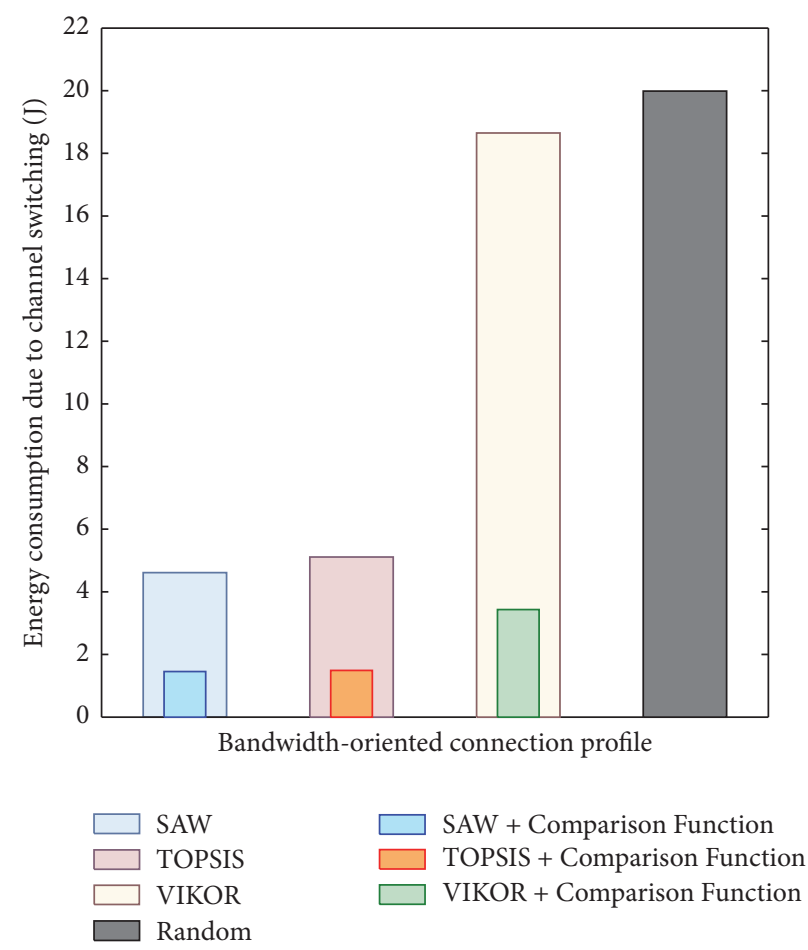

(b) Energy consumption due to channel switching

FIGURE 5: Average performance of MADM algorithms, MADM algorithms combined with Comparison Function of the EMSD and a random case for a bandwidth-oriented connection profile.

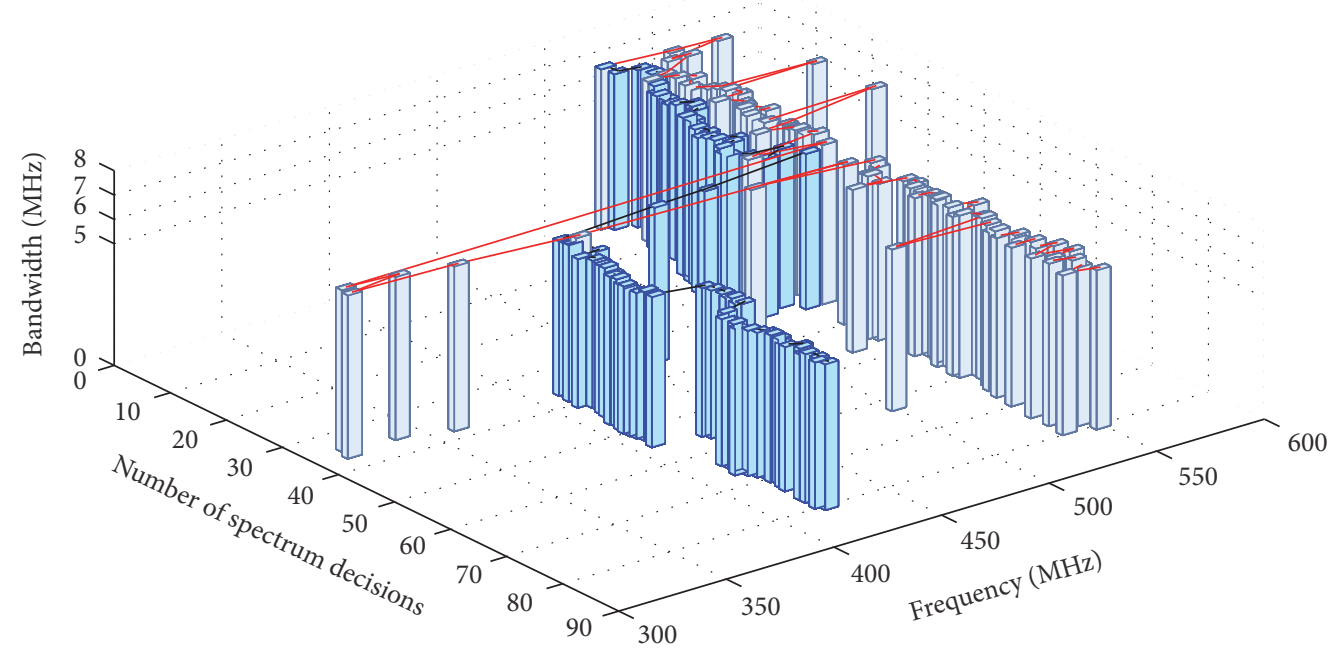

SAW

SAW + Comparison Function

FIGURE 6: Frequency distribution and bandwidth size of the selected spectrum holes by SAW and SAW combined with Comparison Function of the EMSD for 80 decisions.

includes the Comparison Function can save an important amount of energy.

In both Tables 1 and 2, a small reduction of the average bandwidth with the three MADM algorithms is shown when the Comparison Function from our EMSD proposal is used.
In order to show this issue and to appreciate the possible QoS impact that a SU's connection can experience, Figure 7 presents the data rate and energy consumption for SAW (alone) and SAW combined with the Comparison Function for a bandwidth-oriented connection profile. According to 

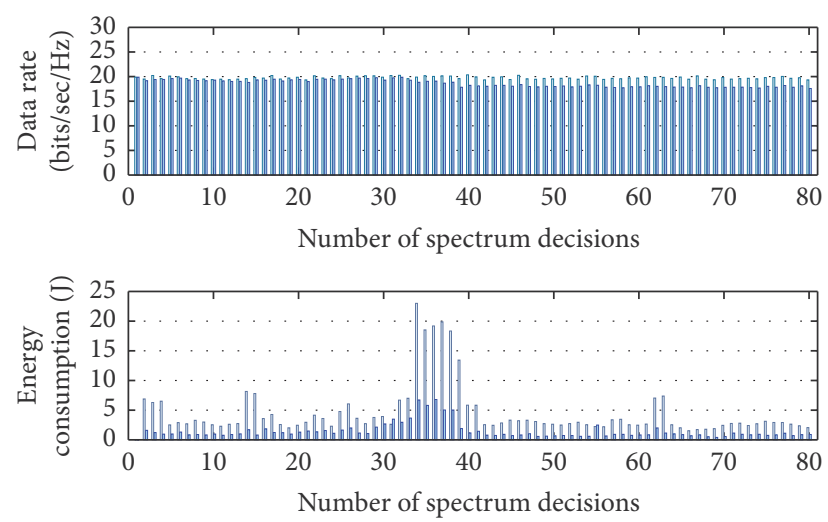

SAW

SAW + Comparison Function

FIGURE 7: Data rate and energy consumed by SAW and SAW combined with Comparison Function of the EMSD for 80 decisions.

[34], the standard IEEE 802.22 considers a data rate between 0.5 and $5 \mathrm{bits} / \mathrm{sec} / \mathrm{Hz}$. The data rate is directly proportional to the size of the bandwidth. Thus, in Figure 7 an estimated average data rate of $3 \mathrm{bits} / \mathrm{sec} / \mathrm{Hz}$ is considered. Also, this figure shows the energy consumption per decision by both algorithm options. In most of the decisions, the data rate is almost the same for SAW and SAW plus the Comparison Function. However, in several decisions SAW with the Comparison Function shows a data rate reduction between $5 \%$ and $10 \%$. Nevertheless, the energy consumed by the Comparison Function in all the spectrum decision is considerably lower compared with SAW (alone), and the percentage of energy consumed is of at least $50 \%$ less per decision. For the scenario in Figure 7, the spectrum holes selected by SAW combined with the Comparison Function achieve almost the same data rate. Nonetheless, the energy consumed by the Comparison Function makes it widely recommendable.

Finally, Figure 8 shows the percentage of the total ECCS saved with the MADM algorithms combined with the Comparison Function in our EMSD proposal. Connection profiles bandwidth and similar weights save from $60 \%$ to $90 \%$ of the ECCS. Also, results of VIKOR have improved for both connection profiles by using the Comparison Function. For the three MADM algorithms considered, it is shown that by following our EMSD proposal their performance can be satisfactory while trading off energy consumption. The proposed EMSD is able to reduce the number of spectrum handoffs considering the ECCS, which significantly improves the overall spectrum decision process in the forthcoming CRNs.

\section{Conclusions}

The aim of this investigation was to reduce the energy switching consumption due to handoffs in CRNs by studying the performance of MADM algorithms in the context of spectrum decision using real spectrum measurements. Thus, an enhanced MADM-based decision functionality was

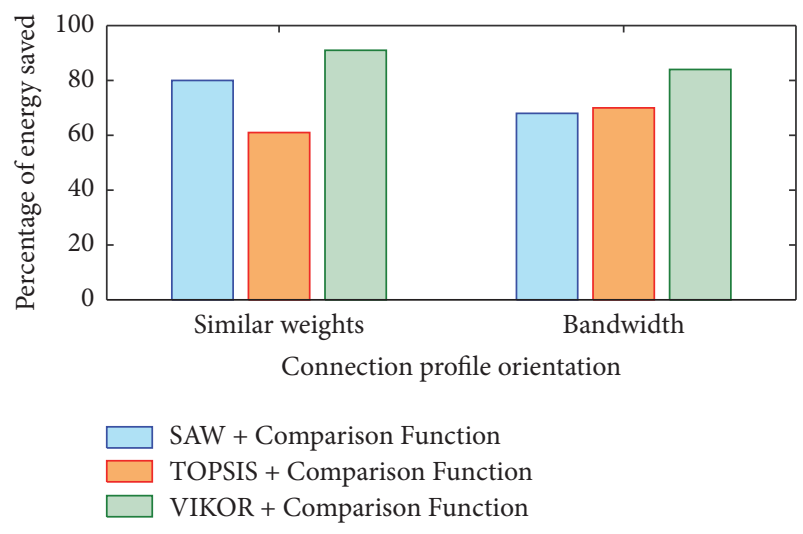

FIGURE 8: Energy saved by using the MADM algorithms combined with Comparison Function of the EMSD regarding MADM algorithms for two different connection profiles.

proposed by taking as inputs the spectrum information of the SUs surrounding environment and its connection requirements. Hence, six decision parameters were considered to characterize each available spectrum hole. Then, these were extracted from real power spectrum measurements and used as inputs of the new MADM-based spectrum decision. It was shown that the proposed spectrum decision algorithm can reduce the number of handoffs by trading off the ECCS. Results quantified savings from $30 \%$ to $90 \%$ in ECCS and reduced number of spectrum handoffs from $47 \%$ to $90 \%$ in TV bands using two different connection profiles.

\section{Competing Interests}

The authors declare that there is no conflict of interests regarding the publication of this manuscript.

\section{Acknowledgments}

This work was supported by the Centre for International Mobility (CIMO) fellowships programme and Centre for Wireless Communications (CWC), Finland. Also, it was supported by the Mexican National Council of Science and Technology (CONACYT), Grant (no. PDCPN2013-01/215499), Universidad Autónoma de San Luis Potosí (UASLP), Mexico, Grant (no. C16-FAI-09-51.51), and Instituto Tecnológico de Sonora (ITSON), Mexico, Grant (no. PROFAPI_2016_0050).

\section{References}

[1] I. F. Akyildiz, W.-Y. Lee, M. C. Vuran, and S. Mohanty, "Next generation/dynamic spectrum access/cognitive radio wireless networks: a survey," Computer Networks, vol. 50, no. 13, pp. 2127-2159, 2006.

[2] I. F. Akyildiz, W.-Y. Lee, M. C. Vuran, and S. Mohanty, "A survey on spectrum management in cognitive radio networks," IEEE Communications Magazine, vol. 46, no. 4, pp. 40-48, 2008.

[3] G. Gür and F. Alagoüz, "Green wireless communications via cognitive dimension: an overview," IEEE Network, vol. 25, no. 2, pp. 50-56, 2011. 
[4] A. P. Bianzino, C. Chaudet, D. Rossi, and J.-L. Rougier, "A survey of green networking research," IEEE Communications Surveys and Tutorials, vol. 14, no. 1, pp. 3-20, 2012.

[5] S. Bayhan and F. Alagöz, "Scheduling in centralized cognitive radio networks for energy efficiency," IEEE Transactions on Vehicular Technology, vol. 62, no. 2, pp. 582-595, 2013.

[6] L. Wang, C. Wang, and C. Chang, "Modeling and analysis for spectrum handoffs in cognitive radio networks," IEEE Transactions on Mobile Computing, vol. 11, no. 9, pp. 1499-1513, 2012.

[7] S. Zahed, I. Awan, and A. Cullen, "Analytical modeling for spectrum handoff decision in cognitive radio networks," Simulation Modelling Practice and Theory, vol. 38, pp. 98-114, 2013.

[8] K. P. Yoon and C.-L. Hwang, Multiple Attribute Decision Making: An Introduction, Sage, 1995.

[9] E. Stevens-Navarro and V. W. S. Wong, "Comparison between vertical handoff decision algorithms for heterogeneous wireless networks," in Proceedings of the IEEE 63rd Vehicular Technology Conference (VTC-Spring '06), vol. 2, pp. 947-951, May 2006.

[10] R. Tawil, G. Pujolle, and O. Salazar, "A vertical handoff decision scheme in heterogeneous wireless systems," in Proceedings of the IEEE 67th Vehicular Technology Conference (VTC '08), pp. 2626-2630, May 2008.

[11] J. D. Martínez-Morales, U. Pineda-Rico, and E. StevensNavarro, "Performance comparison between MADM algorithms for vertical handoff in $4 \mathrm{G}$ networks," in Proceedings of the IEEE 7th International Conference on Electrical Engineering, Computing Science and Automatic Control (CCE '10), pp. 309314, Tuxtla Gutierrez, Mexico, September 2010.

[12] E. Rodriguez-Colina, P. C. Ramirez, and C. E. Carrillo A, "Multiple attribute dynamic spectrum decision making for cognitive radio networks," in Proceedings of the 8th IEEE and IFIP International Conference on Wireless and Optical Communications Networks (WOCN '11), pp. 1-5, May 2011.

[13] R. Aguilar-Gonzalez, M. Cardenas-Juarez, U. Pineda-Rico, and E. Stevens-Navarro, "Performance of MADM algorithm with real spectrum measurements for spectrum decision in cognitive radio networks," in Proceedings of the IEEE 11th International Conference on Electrical Engineering, Computing Science and Automatic Control (CCE '14), pp. 1-6, September 2014.

[14] R. Aguilar-Gonzalez, M. Cardenas-Juarez, U. Pineda-Rico, and E. Stevens-Navarro, "Spectrum occupancy measurements below $1 \mathrm{GHz}$ in the city of San Luis Potosi, Mexico," in Proceedings of the IEEE 78th Vehicular Technology Conference (VTC '13), pp. 1-5, Las Vegas, Nev, USA, September 2013.

[15] F. H. P. Fitzek and M. D. Katz, Mobile Clouds: Exploiting Distributed Resources in Wireless, Mobile and Social Networks, John Wiley \& Sons, Chichester, UK, 2014.

[16] T.-M. Grønli, J. Hansen, G. Ghinea, and M. Younas, "Every cloud has a push data lining: incorporating cloud services in a context-aware application," Mobile Information Systems, vol. 2015, Article ID 278713, 10 pages, 2015.

[17] S.-H. Wu, H.-L. Chao, C.-H. Ko et al., "A cloud model and concept prototype for cognitive radio networks," IEEE Wireless Communications, vol. 19, no. 4, pp. 49-58, 2012.

[18] J. J. Lehtomäki, M. López-Benítez, K. Umebayashi, and M. Juntti, "Improved channel occupancy rate estimation," IEEE Transactions on Communications, vol. 63, no. 3, pp. 643-654, 2015.

[19] K. B. Letaief and W. Zhang, "Cooperative communications for cognitive radio networks," Proceedings of the IEEE, vol. 97, no. 5, pp. 878-893, 2009.
[20] M. Lõpez-Benítez and F. Casadevall, "Methodological aspects of spectrum occupancy evaluation in the context of cognitive radio," European Transactions on Telecommunications, vol. 21, no. 8, pp. 680-693, 2010.

[21] M. H. Islam, C. L. Koh, and S. W. Oh, "Spectrum survey in singapore: occupancy measurements and analyses," in Proceedings of the International Conference on Cognitive Radio Oriented Wireless Networks and Communications (CrownCom '08), May 2008.

[22] Radiocommunication-Bureau, "HANDBOOK spectrum monitoring," Tech. Rep., International Telecommunications Union (ITU), 2002.

[23] R. Zhang, M. Wang, L. X. Cai, Z. Zheng, X. S. Shen, and L.-L. Xie, "LTE-unlicensed: The future of spectrum aggregation for cellular networks," IEEE Wireless Communications, vol. 22, no. 3, pp. 150-159, 2015.

[24] W. J. Hillery, N. Mangalvedhe, and R. Bartlett, "A network performance study of LTE in unlicensed spectrum," in Proceedings of the Globecom Workshops, December 2015.

[25] M. Sharma, A. Sahoo, and K. D. Nayak, "Channel selection under interference temperature model in multi-hop cognitive mesh networks," in Proceedings of the 2nd IEEE International Symposium on New Frontiers in Dynamic Spectrum Access Networks (DySPAN '07), pp. 133-136, Dublin, Ireland, April 2007.

[26] T. C. Clancy, "Formalizing the interference temperature model," Wireless Communications and Mobile Computing, vol. 7, no. 9, pp. 1077-1086, 2007.

[27] W. Zhang, "Handover decision using fuzzy MADM in heterogeneous networks," in Proceedings of the IEEE Wireless Communications and Networking Conference (WCNC '04), vol. 2, pp. 653-658, Atlanta, Ga, USA, March 2004.

[28] E. Stevens-Navarro, R. Gallardo-Medina, U. Pineda-Rico, and J. Acosta-Elias, "Application of MADM Method VIKOR for vertical handoff in heterogeneous wireless networks," IEICE Transactions on Communications, vol. 95, no. 2, pp. 599-602, 2012.

[29] G. Cheng, W. Liu, Y. Li, and W. Cheng, "Joint on-demand routing and spectrum assignment in cognitive radio networks," in Proceedings of the IEEE International Conference on Communications (ICC '07), pp. 6499-6503, Glasgow, UK, June 2007.

[30] S. Krishnamurthy, M. Thoppian, S. Venkatesan, and R. Prakash, "Control channel based MAC-layer configuration, routing and situation awareness for cognitive radio networks," in Proceedings of the Military Communications Conference (MILCOM '05), Atlantic City, NJ, USA, October 2005.

[31] R. Zhang, M. Wang, L. X. Cai, Z. Zheng, X. Shen, and L.-L. Xie, "LTE-unlicensed: the future of spectrum aggregation for cellular networks," IEEE Wireless Communications, vol. 22, no. 3, pp. 150-159, 2015.

[32] A. Jahan, K. L. Edwards, and M. Bahraminasab, Multi-Criteria Decision Analysis for Supporting the Selection of Engineering Materials in Product Design, Butterworth-Heinemann, 2013.

[33] J. Wang, M. S. Song, S. Santhiveeran et al., "First cognitive radio networking standard for personal/portable devices in TV white spaces," in Proceedings of the IEEE Symposium on New Frontiers in Dynamic Spectrum, pp. 1-12, April 2010.

[34] C. R. Stevenson, G. Chouinard, Z. Lei, W. Hu, S. J. Shellhammer, and W. Caldwell, "IEEE 802.22: the first cognitive radio wireless regional area network standard," IEEE Communications Magazine, vol. 47, no. 1, pp. 130-138, 2009. 

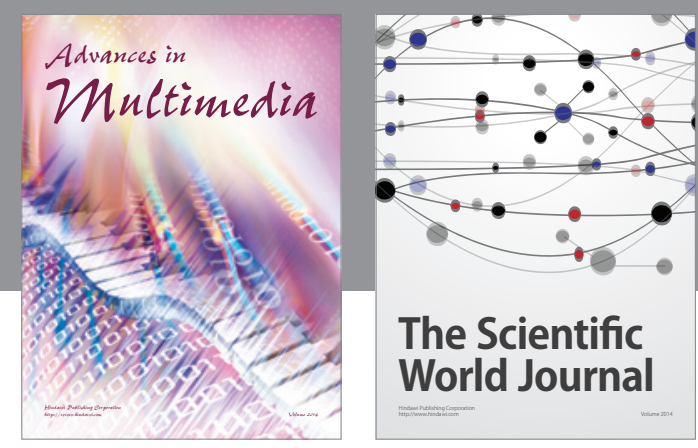

The Scientific World Journal
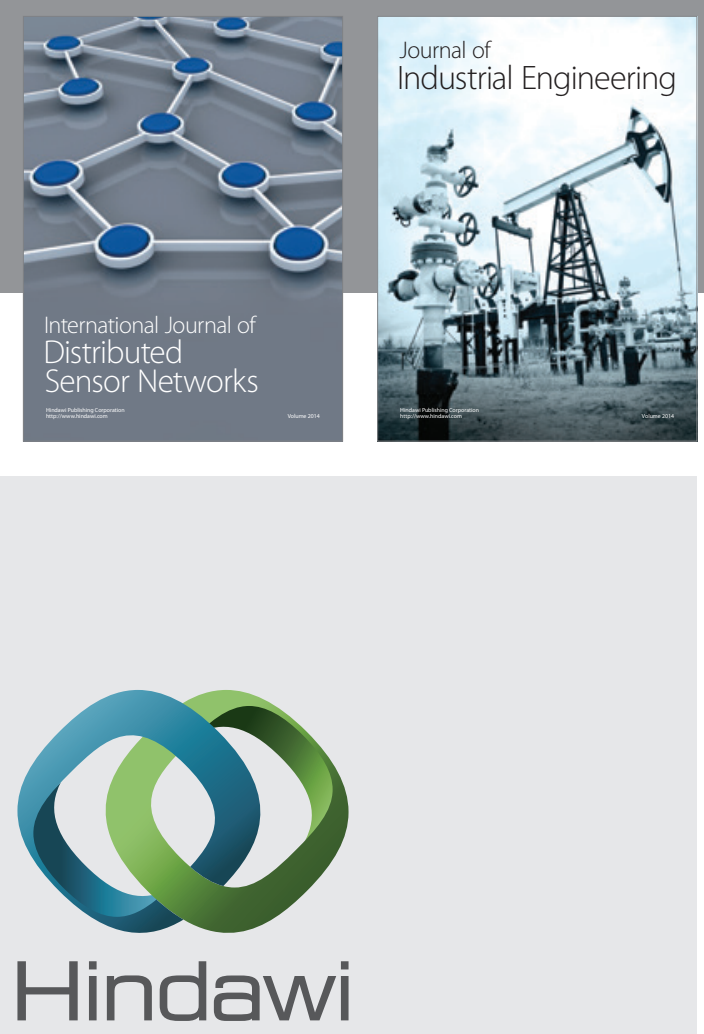

Submit your manuscripts at

http://www.hindawi.com

\section{Computer Networks} and Communications
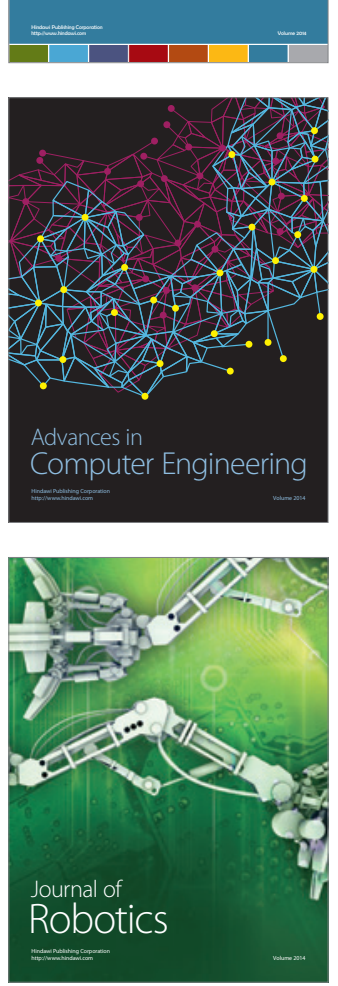
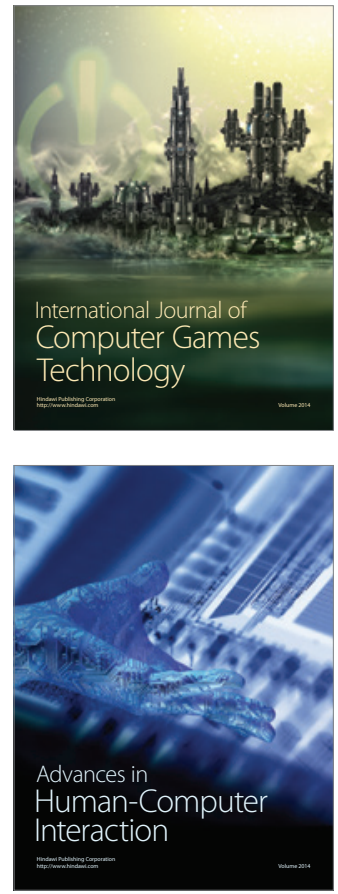
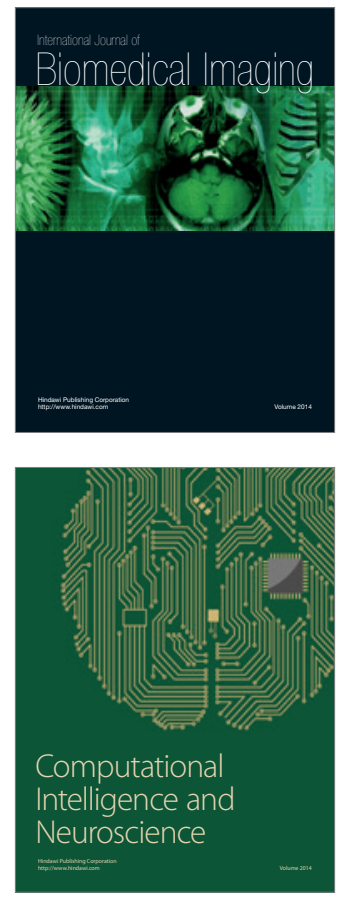
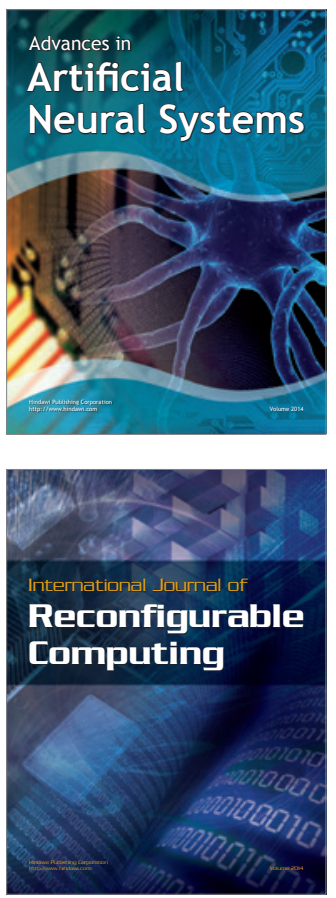
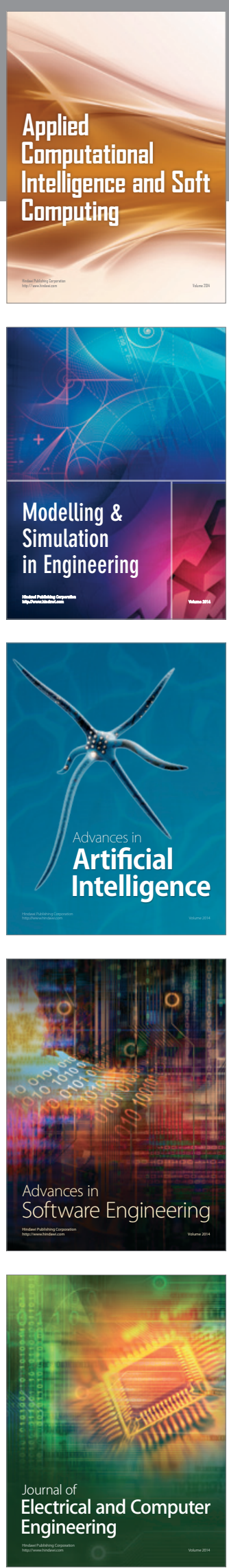